\title{
Transitional Care for Patients with Inflammatory Bowel Disease: Japanese Experience
}

\author{
Hideki Kumagai ${ }^{a}$ Yasuo Suzuki ${ }^{b}$ Toshiaki Shimizuc \\ aDepartment of Pediatrics, Jichi Medical University, Shimotsuke, Japan; ${ }^{b}$ Inflammatory Bowel Disease Center, \\ Toho University Sakura Medical Center, Sakura, Japan; 'Department of Pediatrics, Juntendo University Faculty of \\ Medicine, Bunkyo, Japan
}

\section{Keywords}

Adolescent · Inflammatory bowel disease · Joint clinic ·

Transition · Transitional care

\begin{abstract}
Background: Transition-related healthcare intervention has recently been receiving worldwide attention. Given the increasing incidence of pediatric inflammatory bowel disease (IBD) and its lifelong impact, transitional care has become an important issue. In Japan, guidelines to support the autonomy of IBD patients during transition were recently published.

Summary: Here, we review current issues regarding care for IBD patients during transition from the pediatric to adult period in order to identify the barriers and key elements for successful transition in the context of the Japanese system. Although no single optimal model of transitional care exists, crucial elements identified so far include a joint pediatric/ adult clinic or alternating visits between pediatric and adult healthcare providers, a multidisciplinary approach, and good coordination among stakeholders. Self-reliance and independence of patients with childhood-onset IBD are also considered essential for successful transition. Various tools for assessment of transition readiness have been validated and are considered useful. Better outcomes are expected for individually tailored transition, including improvements in
\end{abstract}

medication adherence, perceived health status, quality of life, and self-management. The timing of transfer from a pediatric to an adult gastroenterologist should not be fixed because the issue is not chronological age but rather the degree of individual maturity. We also propose a standardized medical summary with a checklist template for official referral of patients from a pediatric to an adult gastroenterologist. Key Messages: Transition programs require a multidisciplinary approach with a coordinator (IBD nurse) and optimal collaboration and communication. Lack of resources and funding are also pertinent issues.

(c) 2020 S. Karger AG, Basel

\section{Introduction}

In Japan, transition-related healthcare intervention is a relatively new medical concept that has been receiving attention only since around 2010, behind North America or Europe. This can be defined as the "purposeful planned movement of adolescents and young adults with chronic physical and medical conditions from child-centered to adult-oriented healthcare systems" [1]. Inflammatory bowel disease (IBD; Crohn's disease, ulcerative colitis, and inflammatory bowel disease unclassified) is a chronic relapsing inflammatory disorder of the intestine, with

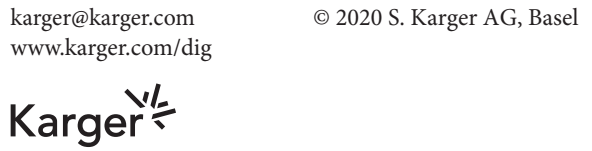


an increasing incidence and prevalence globally, including Japan [2,3]. In 2013, the prevalence of Japanese pediatric Crohn's disease and ulcerative colitis was 7.2 in 100,000 and 15.0 in 100,000, respectively [3]. IBD is diagnosed before the age of 18 years in approximately $25 \%$ of all patients; approximately one-quarter of all affected children and adolescents are under the age of 10 years at diagnosis [4]. In other words, IBD occurs at a delicate time in a child's physical and psychosocial development and can impact on education, employment, and social integration [5]. Deficiencies in transitional care during a particularly critical phase can result in treatment interruptions, insufficient treatment adherence, and an increased frequency of complications that are probably avoidable [6]. Given the increasing incidence of childhood-onset IBD and the lifelong impact of the disease, transitional care for affected patients is critical to ensure continuity of the care and promote health literacy [5]. There is broad consensus that the transition process ought to be well coordinated with the support of a multidisciplinary team [7].

This article reviews current knowledge of transitional care for IBD patients in the context of the situation in Japan. The authors also propose a standardized transfer checklist for smooth official referral from a pediatric gastroenterologist to an adult gastroenterologist.

\section{Methods}

A literature search on PubMed was conducted covering the period from January 2010 to April 2020, using the keywords "inflammatory bowel disease" and "transition." This search brought up a total of 398 relevant articles. After screening the titles, the authors went through the articles and excluded those that were irrelevant. Secondary data sources were found through a bibliographic review of the selected articles. Handsearching of themes related to pregnancy was also conducted. The pertinent points were then summarized.

\section{Current Issues Related to Transitional Care}

There are some instances where childhood-onset IBD patients continue to be treated by pediatric healthcare providers even after reaching maturity, for example, when faced with medical issues unique to adulthood, such as lifestyle-related diseases (atherosclerosis, heart disease, etc.) and malignant neoplasms, especially when they have an increased risk of cancer secondary to longstanding intestinal inflammation or those secondary to immunosuppressive therapies [8]. Many concerns among IBD patients exist regarding impact of the disease during pregnancy. Internists and obstetricians/gynecologists can appropriately address these conditions, whereas pediatricians cannot $[9,10]$. On the other hand, adult gastroenterologists may not be familiar with vaccination issues or conditions associated with treatment using steroids, immunomodulatory agents, or biologics during periods of growth, and thus, the choice of treatments for childhoodonset IBD may be different from that of adults [11]. In addition, guardians often have a high level of trust in their pediatricians, not only for medical care but also for advices regarding social problems. As a result, patients and/ or parents often hesitate to establish new relationships with adult healthcare providers. Patients with childhoodonset IBD require long-term outpatient care and/or hospitalization, and as a result, there may often be delayed psychological and social development or lack of self-care management involving drug adherence.

\section{Endorsement from Organizations}

In 2013, the Japan Society of Pediatrics published a consensus statement on healthcare transition for young adults with special healthcare needs [12]. The statement emphasized that the goal of transitional healthcare is to provide appropriate and uninterrupted healthcare services as the individual moves from adolescence to adulthood and to maximize lifelong functioning. It also states the goal is not always transfer to adult healthcare providers. Based on the patient's physical and/or intellectual disability, transfer from a pediatric healthcare system to an adult one may not be completely achieved.

Transition process itself is an important step allowing gradual adjustment according to the age and maturity of the patient. Structured transition has been endorsed by major international gastroenterology societies, including North American Society for Pediatric Gastroenterology, Hepatology, and Nutrition (NASPGHAN) and European Crohn's and Colitis Organization (ECCO) $[7,13]$. The ECCO considers that IBD patients participating in a transition program are likely to have better adherence and less adverse outcomes after transfer to adult healthcare provider compared with those who do not become involved. In Japan, the Japanese Society for Pediatric Gastroenterology, Hepatology, and Nutrition (JSPGHAN) recently published guidelines on autonomy support for patients with IBD during the transitional period [14]. 


\section{Stakeholders in Transposal Care}

The transitional care for young IBD patients involves multiple stakeholders (i.e., parents/caregivers, school workers, nurses, dietitians, counselors, social workers, psychologists, healthcare systems, insurance providers, and pharmacists) whose roles are all essential for seamless continuation of an adolescent's healthcare. Recognition of the roles of these individuals in multidisciplinary care and maximization of communication between them is of strategic importance [15-18]. In particular, specialized IBD nurses are expected to coordinate various issues among pediatric healthcare providers, adult healthcare providers, patients, and the guardians and play an important role in multidisciplinary IBD unit for transitional care $[17,19]$. Patients with IBD managed by a transition coordinator show significant better transition readiness and acquisition of self-management skills [20]. In China, IBD nurses provide instructions to patients in the hospital, visit patients at home, maintain social networking groups, and make phone calls to promote follow-up [21]. However, in the Japanese survey, poor coordination of care was identified [16].

\section{Supporting Tools for Transition}

It is considered that using transition readiness checklists and handouts are useful for clarification of patient's goal and regular assessment of his/her present level of achievement. The abovementioned Japanese Guideline for Autonomy Support of Patients with IBD includes a checklist of self-management skills for patients, a transition readiness checklist for guardians, educational materials for young teenagers regarding the digestive anatomy and physiology, a rough standard schedule for transfer to adult gastroenterologist, a template for patient's own medical record, and "path" which includes the time schedule of transition for each of the multidisciplinary professionals involved [14]. It is expected that using these tools will lead to a better understand of each patient's status and make it easier to confirm the goals of not only the patient and guardians but also each professional care provider. However, validation of the guideline has not yet been evaluated. Other tools include Transition Readiness Assessment Questionnaire and the NASPGHAN checklist. The former consists of a 20-item self-report questionnaire regarding disease self-management skills [22]. It covers a range of knowledge and skills likely related to a patient's ability to transition from pediatric-centered to adult healthcare, but it is not specified for IBD and is based on self-reporting without clinical correlation. The latter checklist aims to be a reminder of how and when to encourage IBD patients to take on more responsibility for a successful transition [23]. Other IBD-specified tools include the Short IBD Questionnaire [24], the IBD-yourself [25], and the "IBD Self-Efficacy Scale for Adolescents and Young Adults," which involves a 13-item disease-specific scale designed to aid in managing medical care, everyday life with IBD, feedings, and living with IBD in the future [26].

\section{Barriers to Successful Transition}

Many previous studies have identified various barriers to a seamless transfer of care, as shown in Table $1[5,13$, $16,18,19,27]$. These cover a range of specialists such as healthcare providers, patients, guardians, and social systems and may be single or multiple. Recent and current development of guidelines and consensus statements suggests that awareness of providing good transitional care is developing on the basis of identified barriers.

\section{Transitional Care and Pregnancy}

Few studies have investigated pregnancy in patients with childhood-onset IBD in terms of transitional care, and limited information is available on the lifestyle of such patients. However, it is considered important to educate them or address sexuality and fertility issues with patients in transitional period, prior to any pregnancy. According to the Healthcare Provider Transitioning Checklist by NASPGHAN [23], providing information about sexuality should be started at the age of 12-14 years and discussion about the impact of IBD on sexuality and fertility should be started at the age of 14-17 years. The conversation should include details of self-prescribed diets and other interventions, such as steroids, immunomodulatory agents, and biologics, which may affect not only the patients but also their fetuses. On the other hand, it is reported that many women with IBD remain childless due merely to lack of information under the assumption that pregnancy would be medically dangerous and that parenting would be too challenging in addition to their illness [28]. It is recommended that pediatric healthcare professionals begin to provide information about the impact of IBD on reproduction and to start any conversation in the transitional period. Pregnancy in relation to IBD requires a holistic and multidisciplinary approach. 
Table 1. Barriers to successful transition

\section{Patient}

Psychological immaturity, delay of independence

Disease non-remission (active phase)

Emotional/cognitive delay

Attachment to pediatric healthcare providers, medical setting, or practice

Lack of knowledge about disease concept and own medical history

Unstable social situation

Presence of diseases other than IBD

\section{Caregiver}

High parental involvement

Emotional/cognitive issue

Lower socioeconomic status

Underestimation of the child's capability

Reduced parental assistance

Attachment to pediatric healthcare providers, medical setting, or practice

Pediatric healthcare provider

Attachment to patient or family

Inadequate medical summary

Poor care coordination with adult gastroenterologist

Lack of manpower or time to prepare for transition

Adult healthcare provider

Reluctance of patients and parents due to their close relationship with pediatric healthcare provider

Cultural difference from pediatric healthcare providers

Poor care coordination with pediatric gastroenterologist

Lack of awareness of importance of transitional care

Health system organization

Lack of defined transition protocol (manual), planning difficulties

Lack of manpower (e.g., coordinator, IBD nurse), time/space or funding

IBD, inflammatory bowel disease

\section{Timing of Transfer to an Adult Gastroenterologist}

Transfer from a pediatric to an adult gastroenterologist should ideally take place during the disease remission phase. However, there is no clear cutoff for transfer. The abovementioned Japanese survey indicated that adult gastroenterologists considered an age of 16 years to be reasonable to transfer point [16]. However, another Japanese survey of pediatric gastroenterologists revealed some discrepancy in their views regarding the timing of transfer in comparison to adult gastroenterologists: the majority of the former considered an age of 18 years or above to be ideal [29]. Both surveys showed that complex issues, such as psychological immaturity and autism spectrum disorders, presented a considerable barrier to successful patient transition and might necessitate some delay before transfer. Developmental milestones in adolescence may be reached at different times. Stollon et al. [30] reported that self-management skills were not mastered un- til after 18 years of age. In any event, the timing of transfer should not be fixed because the issue is not one of chronological age but rather when each individual achieves maturity [31].

\section{Joint Clinic and Alternative Visit}

One of the ideal models for support of patients with childhood-onset IBD during the transition period is to implement an outpatient-based transitional care clinic run by a multidisciplinary team [27]. Although there is no common definition of a transition clinic and the settings are notably heterogeneous [17], a multidisciplinary approach and good interaction among the various stakeholders are potential keys of the success of transition [5]. In some circumstances, it may be difficult to set up a transitional clinic in some institutions due to territorial dispersion (regional specifics) or lack of medical resources. 
Table 2. Key elements for successful transition

Good coordination between pediatric and adult healthcare provider

Initiation of planning and education of patients and parents at least 1 year before transfer

Discussion with the patient and caregiver regarding self-management of the disease

Consideration of the patient's sentiment in transition planning

Providing an opportunity to attend the office alone

Setting up a patient's meeting with an adult gastroenterologist before official transfer (joint clinic, alternating visits)

Provision of a medical summary by a pediatric gastroenterologist before the first visit to an adult gastroenterologist

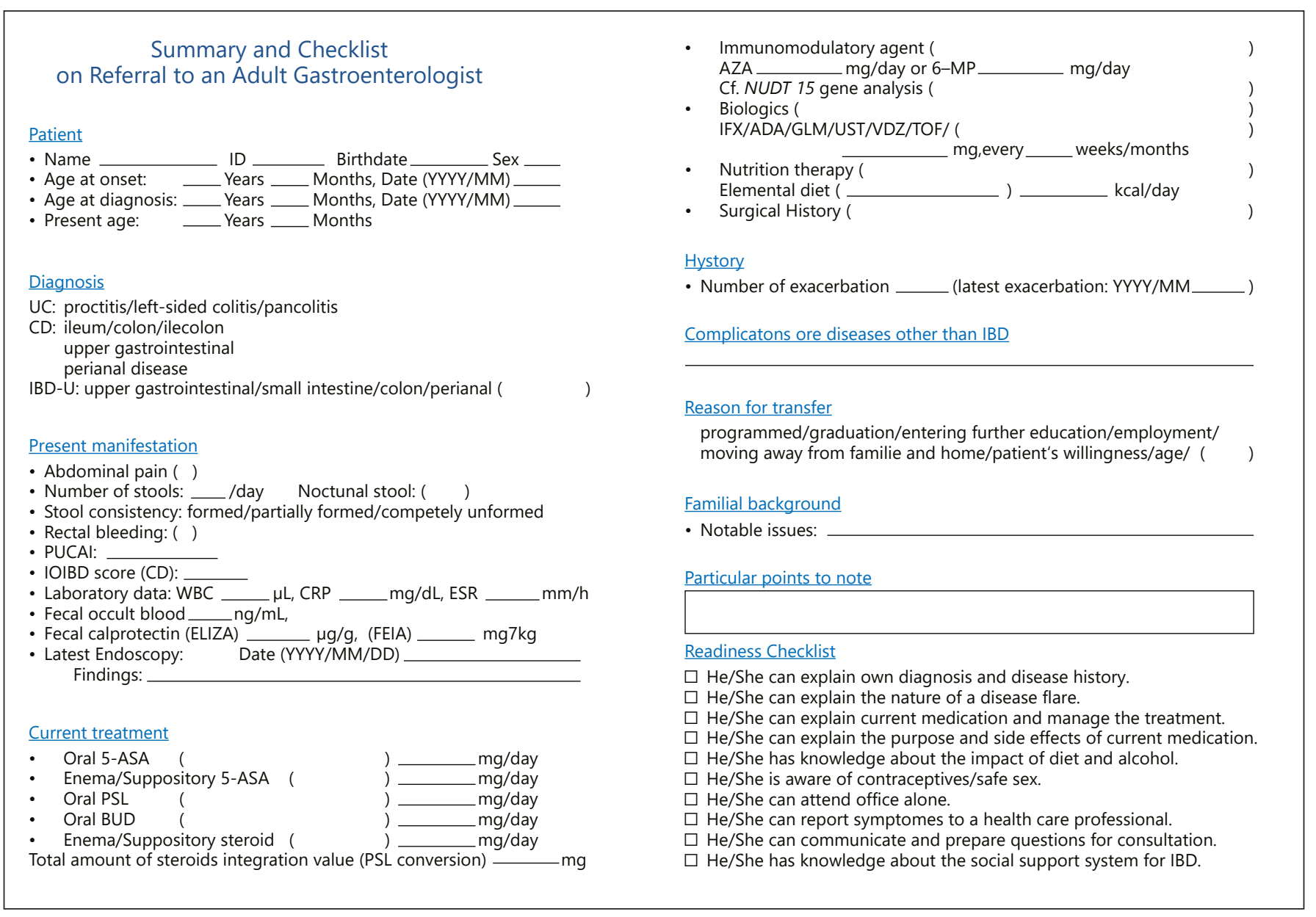

Fig. 1. Proposed template for standardized medical summary with a checklist for referral. UC, ulcerative colitis; $\mathrm{CD}$, Crohn's disease; IBD-U, inflammatory bowel disease unclassified; PUCAI, Pediatric Ulcerative Colitis Activity Index; IBD, inflammatory bowel disease; IOIBD, International Organization for Study of Inflammatory Bowel Disease.

In such cases, alternating visits between pediatric and adult healthcare services or email contacts may be useful approaches for encouraging patient autonomy and individual decision-making while still providing some reassuring support in the familiar background of the pediatric clinic $[13,17,18,20]$.

\section{Key Elements for a Successful Transition}

An international Delphi study conducted by experts of transitional care from 15 countries, including physicians, nurses, psychologists, transition program managers, and research director, presented key elements for, 
and indicators of, a successful transition [32]. Similarly, a multinational expert panel made up of IBD nurses and pediatric and adult gastroenterologists investigated the outcomes that reflect successful transition in IBD patients using a Delphi method [33]. The principal key elements are shown in Table 2. The important points for successful transition are adequate teaching about the concept of transition to patients and guardians, early planning, and starting the program before it becomes too late. Of course, the ideal form of intervention involves a multidisciplinary team of specialized professionals. Moreover, it is very important to regularly monitor the transition process for both the patient and provider, in order to optimize and individualize the outcome. Transition takes time and should be personalized according to the needs, personality, and clinical history of the patients involved [5].

On official transfer from a pediatric to an adult gastroenterologist, proper provision of the patient's medical record to the latter was one of the most important points highlighted by the abovementioned Japanese surveys [16, 29]. JSPGHAN has presented a standardized template for the medical record which is also designed to allow the patient to be able to manage alone [14].

\section{Proposal of a Standardized Medical Summary \& Checklist for Referral}

Poor coordination between pediatric and care providers and provision of an inadequate medical summary are known to be barriers to smooth transition $[16,29]$. Therefore, provision of a proper medical record documented by a pediatric gastroenterologist is essential. However, redundant and pointless entries may be a source of irritation to an adult gastroenterologist. For this reason, the authors propose a standardized template for the medical summary and a checklist for eventual referral from a pediatric healthcare provider to an adult gastroenterologist (Fig. 1).

\section{Conclusion}

Due to differences in medical environments, resources, and culture, various societies require different levels of intensity for an IBD transition program. Transition programs should be tailored to and meet the individualized needs of patients that are identified through discussions with the patients and their families about their expecta- tions and fears about transition. Better outcomes are expected from individually tailored transition planning, including improvements in medication adherence, perceived health status, quality of life, and self-management. Transition programs in Japan require a multidisciplinary approach with a coordinator (IBD nurse), thus improving collaboration and communication. Other issues that need to be discussed would include lack of resources and funding relevant to the cost-benefit balance during transitional care.

\section{Acknowledgement}

This study was supported in part by Health and Labor Sciences Research Grants for studies on intractable diseases from the Ministry of Health, Labor, and Welfare of Japan (29FC1037).

\section{Conflict of Interest Statement}

The authors have no conflicts of interest to declare.

\section{Funding Sources}

This study was supported in part by Health and Labor Sciences Research Grants for studies on intractable diseases from the Ministry of Health, Labor, and Welfare of Japan (29FC1037).

\section{Author Contributions}

H.K. designed and drafted the manuscript; T.S. and Y.S. critically reviewed the manuscript. All authors read and approved the final manuscript.

\section{References}

1 Blum RW, Garell D, Hodgman CH, Jorissen TW, Okinow NA, Orr DP, et al. Transition from child-cantered to adult health-care systems for adolescents with chronic conditions. a position paper of the society for adolescent medicine. J Adolesc Health. 1993;14:570-6.

2 Baumgart DC, Bernstein CN, Abbas Z, Colombel JF, Day AS, D'Haens G, et al. IBD around the world: comparing the epidemiology, diagnosis, and treatment: proceedings of the world digestive health day 2010-inflammatory bowel disease task force meeting. Inflamm. Bowel Dis. 2011;17:639-44.

3 Ishige T, Tomomasa T, Hatori R, Tatsuki M, Igarashi Y, Sekine K, et al. Temporal trend of pediatric inflammatory bowel disease: analysis of national registry data 2004-2013 in Japan. J Pediatr Gastroenterol Nutr. 2017;65(4): e80-2. 
4 Benchimol EI, Fortinsky KJ, Gozdyra P, van den Heuvel M, van Limbergen J, Griffiths AM. Epidemiology of pediatric inflammatory bowel disease: a systematic review of international trends. Inflamm Bowel Dis. 2011;17(1): 423-39.

5 Nardone OM, Iacucci M, Ghosh S, Castiglione F. Can a transition clinic bridge the gap between paediatric and adult inflammatory bowel disease care models? Dig Liver Dis. 2020;52(5):516-27.

6 van Staa AL, Jedeloo S, van Meeteren J, Latour JM. Crossing the transition chasm: experiences and recommendations for improving transitional care of young adults, parents and providers. Child Care Health Dev. 2011;37(6): 821-32.

7 Baldassano R, Ferry G, Griffiths A, Mack D, Markowitz J, Winter H. Transition of the patient with inflammatory bowel disease from pediatric to adult care: recommendations of the north american society for pediatric gastroenterology, hepatology and nutrition. J Pediatr Gastroenterol Nutr. 2002;34(3):245-8.

8 Axelrad JE, Lichtiger S, Yajnik V. Inflammatory bowel disease and cancer: the role of inflammation, immunosuppression, and cancer treatment. World J Gastroenterol. 2016; 22(20):4794-801.

9 Ariyasu H, AkamizuCurrent Status T, Regarding I. Current status and issues regarding transitional health care for adults and young adults with special health care needs in Japan. Intern Med. 2018;57(10):1337-44.

10 Restellini S, Biedermann L, Hruz P, Mottet C, Moens A, Ferrante M, et al. Update on the management of inflammatory bowel disease during pregnancy and breastfeeding. Digestion. 2020 Jan 8:1-16.

11 Manser CN, Maillard MH, Rogler G, Schreiner P, Rieder F, Bühler S. Vaccination in patients with inflammatory bowel diseases. Digestion. 2020 Jan 22:1-11.

12 Yokoya S, Ochiai R, Kobayashi N, Komamatsu H, Mashiko T, Mizuguchi M, et al. Recommendations for transitional care for patients with childhood-onset disease. J Jpn Pediatr Soc.. 2014;118:98-106.

13 van Rheenen PF, Aloi M, Biron IA, Carlsen K, Cooney R, Cucchiara S, et al. European crohn's and colitis organisation topical review on transitional care in inflammatory bowel disease. J Crohns Colitis. 2017;11(9):1032-8.
14 Kumagai H, Akiyama T, Abukawa D, Ida S, Inui A, Kudo T, et al. Guidelines for autonomy support of patients with IBD in the transitional period. Jpn J Pediatr Gastroenterol Hepatol Nutr. 2018;32:15-27.

15 Afzali A, Wahbeh G. Transition of pediatric to adult care in inflammatory bowel disease: is it as easy as 1, 2, 3? World J Gastroenterol. 2017;23(20):3624-31.

16 Kumagai H, Kudo T, Uchida K, Kunisaki R, Sugita A, Ohtsuka Y, et al. Adult gastroenterologists' views on transitional care: results from a survey. Pediatr Int. 2019;61(8):81722.

17 Erôs A, Soós A, Hegyi P, Szakács Z, Erőss B, Párniczky A, et al. Spotlight on transition in patients with inflammatory bowel disease: a systematic review. Inflamm Bowel Dis. 2020; 26(3):331-46.

18 Tan B, Ong D. Pediatric to adult inflammatory bowel disease transition: the asian experience. Intest Res.. 2020;18(1):11-7.

19 Sánchez CS, Hernani MDMT, Calatayud GA, Cid MDCM, López VMN, Jiménez IM, et al. Status of transition care in inflammatory bowel disease in spain. Different medical perspectives. Rev Esp Enferm Dig. 2019;111:8338

20 Gray WN, Holbrook E, Dykes D, Morgan PJ, Saeed SA, Denson LA, et al. Improving IBD transition, self-management, and disease outcomes with an in-clinic transition coordinator. J Pediatr Gastroenterol Nutr. 2019;69(2): 194-9.

21 Zhu X-Q, Li R-X, Chen F. Impact of transitional care on self-management ability and quality of life in patients with inflammatory bowel disease(in Chinese with English abstract. Wcjd. 2016;24(25):3723-8.

22 Sawicki GS, Lukens-Bull K, Yin X, Demars N, Huang I-C, Livingood W, et al. Measuring the transition readiness of youth with special healthcare needs: validation of the traq: transition readiness assessment questionnaire. J Pediatr Psycol. 2011;36:160-71.

23 The Children's Digestive Health \& Nutrition Foundation [Internet]. Transitioning a patient with IBD from pediatric to adult care [Accessed 2020 May 8]. Available from: https://www.naspghan.org/files/documents/ $\mathrm{pdfs} /$ medical-resources/ibd/Checklist_PatientandHealthcareProdiver_TransitionfromPedtoAdult.pdf.
24 Irvine EJ, Zhou Q, Thompson AK. The short inflammatory bowel disease questionnaire: a quality of life instrument for community physicians managing inflammatory bowel disease. ccrpt investigators. canadian crohn's relapse prevention trial. Am J Gastroenterol. 1996;91(8):1571-8.

25 Zijlstra M, De Bie C, Breij L, van Pieterson M, van Staa A, de Ridder L, et al. Self-efficacy in Adolescents with Inflammatory Bowel Disease: A Pilot Study of the "IBD-yourself". A disease specific questionnaire. J Crohns Colitis. 2013;7:e375-85.

26 Izaguirre MR, Taft T, Keefer L. Validation of a self-efficacy scale for adolescents and young adults with inflammatory bowel disease. J Pediatr Gastroenterol Nutr. 2017;65(5):546-50.

27 Sattoe JNT, Peeters MAC, Haitsma J, van Staa A, Wolters VM, Escher JC. Value of an outpatient transition clinic for young people with inflammatory bowel disease: a mixed-methods evaluation. BMJ Open. 2020;10(1): $10 \mathrm{e} 033535$.

28 Selinger CP, Ghorayeb J, Madill A. What factors might drive voluntary childlessness (vc) in women with ibd? Does ibd-specific pregnancy-related knowledge matter? J Crohns Colitis. 2016;10(10):1151-8.

29 Kumagai H, Kudo T, Uchida K, Kunisaki R, Sugita A, Ohtsuka Y, et al. Transitional care for IBD: a survey of Japanese pediatric gastroenterologists. Pediatr int. 2020.

30 Stollon N, Zhong Y, Ferris M, Bhansali S, Pitts B, Rak E, et al. Chronological age when healthcare transition skills are mastered in adolescents/young adults with inflammatory bowel disease. World J Gastroenterol. 2017; 23(18):3349-55.

31 Goodhand J, Hedin CR, Croft NM, Lindsay JO. Adolescents with IBD: the importance of structured transition care. J Crohns Colitis. 2011;5(6):509-19.

32 Suris JC, Akre C. Key elements for, and indicators of, a successful transition: an International Delphi Study. J Adolesc Health. 2015; 56(6):612-8

33 van den Brink G, van Gaalen MAC, de Ridder L, van der Woude CJ, Escher JC. Health care transition outcomes in inflammatory bowel disease: a Multinational Delphi Study. J Crohn Colitis. 2019;13:1163-72. 